\title{
Chapter 1 \\ A Comprehensive Analytical Framework for Risks, Disasters and Crises
}

\subsection{Motives}

Early warning and emergency management is a comprehensive and integrated system that deals with all sorts of emergencies, risks and crises, and involves various sectors of society and government departments. A consistent and holistic approach, however, has previously been lacking in both research and practice. Scholars of natural sciences and social sciences address the subject differently, so do academics and practitioners; and compartmentalization often characterizes different regions, departments and organizations' responses to emergencies. These problems are particularly clearly manifested in the catastrophic winter storm that hit southern China in 2008 .

\subsubsection{Winter Storm in Southern China}

The winter storm in question was a series of heavy snows and low temperature events that started on January 10, 2008 and caused serious social consequences in large parts of southern China. A typical occurrence in a high-risk society, it is significant in the following ways. First, it is an external manifestation of accumulated risk, and like the world's many other extreme weather events caused by global climate change, it points to the trend that disasters have become more common. Second, it reflects the change in risk structure. It has far greater social implications than ordinary natural disasters, and is closely related to political and social processes such as urban-rural dualism and the provision of public services. Yet another event of systemic tension following the SARS outbreak in 2003, it attracted nationwide attention. Finally, its social implications were uncertain and difficult to predict based on previous experiences. 


\subsubsection{Progression of Disaster}

The progression of the winter storm in 2008 followed six independent paths as shown in Fig. 1.1.

(1) The heavy snows caused the collapse of buildings in urban areas and, more worsely, deaths and injuries in rural areas. Some people were displaced and lived a difficult life.

(2) The heavy snows, ice and low temperatures directly affected the crops and the lives of farmers, disrupting the balance in demand and supply of agricultural products and pushing up the cost of living in urban areas.

(3) The heavy snows, ice and low temperatures disrupted road transportation. Numerous passengers heading home for the Spring Festival were stranded, putting more security pressure on the stations and surrounding areas. Freight transportation was also affected, leading to the shortage and rising cost of necessities.

(4) The accumulation of snow on transmission lines led to massive blackouts. As most trains were powered by electricity after railway electrification, power outages disrupted rail services, leaving a large number of passengers stranded. Apart from hardship to passengers, this also increased security pressure on railway stations and surrounding areas. Snow and ice also impeded freight transportation. The impact of power outage on the production and transportation of coal, an important source of electricity, further worsened the power shortage. Industrial production was hampered, causing imbalance in supply and demand and eventually pushing up prices. Moreover, failures in power grids, which are essential to all sectors nowadays, disrupted water supply, telecommunications, and financial services, among others, affecting people's lives and posing a threat to social stability in some disaster-stricken areas.

(5) Rising prices caused by multiple factors made the lives of some people even more difficult.

(6) Difficulties in living and disruptions of public service exerted pressures on the political system, so did social disorder on the social system, and rising prices and disruptions of thermal coal production and transportation on the economic system. Together they resulted in the tension in the entire political, economic and social systems.

\subsubsection{Impacts of the Disaster}

The six paths described above represent in a nonlinear pattern the systemic risks posed by the storm. Such factors as population, environment, technology, and socioeconomic structure affected the spread of risks in a way that made the consequences of the storm unpredictable.

(1) Population: The 2008 winter storm coincided with Chunyun, a travel rush that, beginning 20 years ago, would take place around the time of Spring Festival and 


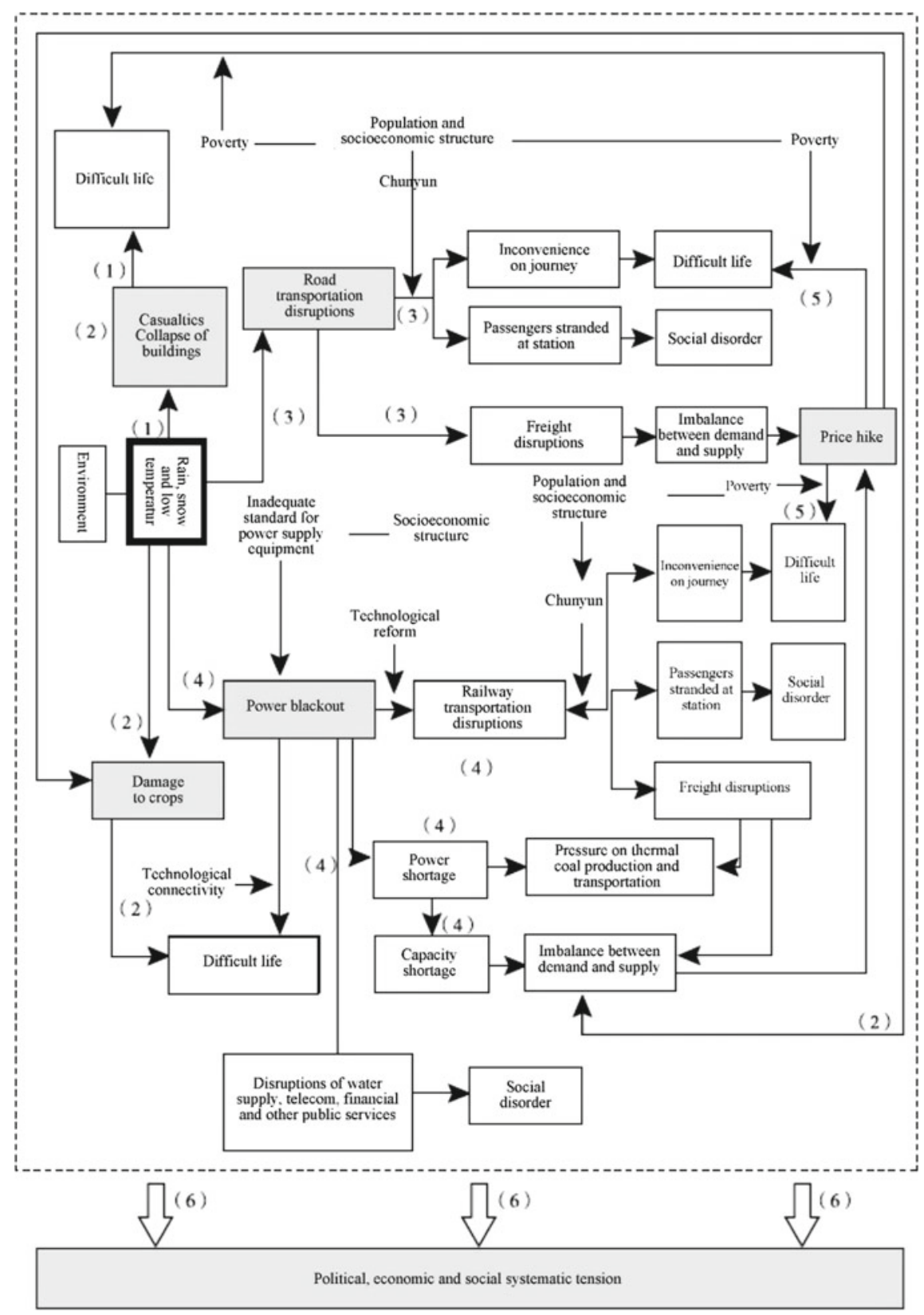

Fig. 1.1 Progression of the 2008 winter storm in southern China and its social impact 
last over 40 days. The travel season saw nearly two billion passengers, including migrant workers, students and other people, travel by sea, air, and land for tour and for family reunion. Apart from busy passenger transportation, freight traffic also peaked.

(2) Environment: The 2008 winter storm, the worst over the past fifty years, included four waves of heavy rain and snow, and was extremely rare in southern China in terms of the sphere of influence, duration, and snowfall intensity. Such extreme weather was a result of global environmental degradation.

(3) Technology: Apart from road closure, the winter storm also caused lengthy delays in rail transportation. This is mainly because electric locomotives had replaced most diesel locomotives due to technological advances and the rail system's dependence on power grids aggravated systemic risks.

(4) Socioeconomic structure: The socioeconomic structure has multi-faceted influence on risk. Chunyun, which was affected by the 2008 winter storm, is typical of China's urban-rural dualism. The failures in power grids were partly attributed to the lack of high-standard power supply equipment, an epitome of structural inadequacy of public spendings in rural areas. The commitment to "bringing every migrant worker back home for family reunion during the Spring Festival" is for the purpose of social integration, a wise decision given the current political, economic and social structures.

\subsubsection{Problems Revealed in Disaster Response}

The winter storm of 2008, the worst over the past 50 years, alarmed us to an array of problems in the social system that had been deemed reasonably structured, wellfunctioning, effectively coordinated and efficiently managed. Factors contributing to these problems include the classification of academic disciplines, division of labor, poor coordination in disaster response, and urban-rural dualism.

\subsubsection{Research on Early Warning and on Emergency Management Are Kept Separate}

Research on the early warning system in society, which is largely confined to the sociological sphere, has long concentrated on risks such as fractured society, social tension and conflicts, but paid insufficient attention to natural disasters and their risks. Likewise, emergency management research, which falls within the scope of administrative management, has highlighted how unexpected crises are and how government responds to them, while ignoring the early phases of natural disasters and their attendant crises. That explained why foresight was lacking in related studies and why neither sociologists nor administrative management scholars warned of the winter storm or provided any contingency plan. 


\subsubsection{Natural Sciences and Social Sciences Treat Disasters Differently}

For long, it is natural sciences and engineering technology that have contributed most to disaster-related discourse and research and management tools, whereas social sciences have played a less important role. In natural-science studies of disasters, the theory of "disaster chain", a chain of disastrous events where one event typically leads to the next, is widely accepted as a basic analytical tool. ${ }^{1}$ The 2008 winter storm, however, posed a challenge to the theory. The existence of hundreds of millions of migrant workers due to China's unique household registration (hukou) system and urban-rural dualism from cities to their rural homes, the large-scale movement of people driven by the tradition of family reunion and the heavy dependence of modern rail transportation on power grids made the "chain effect" of the winter storm more complicated and augmented the disastrous consequences, and these could hardly be explained by natural factors and the linear path of the traditional "disaster chain" theory. In other words, natural disasters are more than "natural"; they also have social attributes, including the social environment where disasters occur and actions taken for disaster relief and mitigation. Obviously, natural sciences alone cannot fully explain the social attributes of disasters.

\subsubsection{Academics and Practitioners Approach Problems Differently}

In general, academics focus on explaining or analyzing problems while practitioners concentrate on solving problems.

Amid the great social changes in China, social problems have increased exponentially, and practitioners' problem-solving mentality can easily cause them to seek the quickest rather than the best solutions. This will inevitably result in short-sightedness in policy formulation. As a matter of fact, research and practice cannot be completely cut off from each other. Now that research is increasingly geared to the practical needs of the country and researchers are warned against the danger of being divorced from reality, it is all-important to carry out forward-looking, systematic and strategic research within a new comprehensive analytical framework. However,

\footnotetext{
${ }^{1}$ As early as the 1980s, Ma Zongjin founded a comprehensive disaster mitigation team, which brought together experts in earthquake, water conservancy, meteorology and agriculture. As an important part of their research output, the team introduced the concept of "disaster chain". Ma put forward three kinds of "disaster chains": "heavy rain (typhoon)-rainstorm-disaster avoidancemudslide-flood-farmland, road and building damage-drowning deaths, etc."; "earthquake-(rain and snow)-collapse-sand surge-damage to buildings (housing, factories, lifeline)-transportation disruptions-communications disruptions-gas leakage, fire, etc."; and "drought-dust storm-heat wave-water shortage-damage to crops, forests and grasses-shrouded rural and urban communities". On this basis, Shi Peijun proposed the "disaster chain" of cold wave: "cold wave-snow disaster (frost, cold temperature)-biological effects of cold and frost (mechanical failure, structural damage, damage to agricultural and pastoral areas)". The theory of "disaster chain" has become one of the main guidelines in the field of natural disaster reduction. See: Ma Zongjin. Some thoughts on emergency response to natural disasters in China. China Emergency Management, 2007 (2); Shi Peijun. Theory and dynamics of disaster research. Journal of Natural Disasters, 2002 (3).
} 
just as academics often criticize practitioners for lack of long-term vision in policy fragmentation, practitioners often accuse academics of rendering no immediate and practical help. A lack of cooperation between them was also shown in their response to the winter storm of 2008 .

\subsubsection{Emergency Response of Different Regions, Departments and Organizations Are Poorly Coordinated}

During the winter storm of 2008, the expressway authority was always the first government department to respond and the action they took was invariably shutting down the roads. While road closure did prevent serious traffic accidents, the closure of the expressway system, which is unprecedently important to our society today, would have a crippling effect on the entire social system-especially after the disruption of the rail network.

Poor coordination also hindered emergency management. For example, the provincial government of Guangdong and the municipal government of Guangzhou were sometimes at odds with China Railway Guangzhou Group, the local arm of the National Railway Administration. They couldn't effectively coordinate their actions and operations, and foot-dragging occurred at times. After the trunk line of the Beijing-Guangzhou railway was closed due to the power grid failure in Chenzhou City of Hunan Province, the provincial government of Guangdong encouraged migrant workers to stay in Guangdong for the Spring Festival instead of returning to their hometown; the local railway authority, however, asked the Guangzhou Railway Station to continue to sell tickets of the Beijing-Guangzhou line. As a result, hundreds of thousands of migrant workers swarmed around the railway station, causing public safety concerns and disturbing the residents nearby. The fact that the provincial government of Guangdong and the municipal government of Guangzhou could not give instructions to the local railway authority and railway station was a hindrance to emergency response efforts.

Noticeably, the winter storm coincided with the "two sessions" in more than ten southern provinces, when the governments, the provincial people's congresses and the provincial committees of the Chinese People's Political Consultative Conference (CPPCC) were holding elections. ${ }^{2}$ China is a typical "government-guided society", where the government leads economic development, and emergency management, too. During the 2008 winter storm, as local governments were busy with the "two sessions" and elections, they can hardly have enough time and effort to cope with the disaster, missing out on the best time for disaster mitigation and relief.

\footnotetext{
${ }^{2}$ Likewise, when Severe Acute Respiratory Syndrome (SARS) swept across Guangdong at the beginning of 2003, the province was holding the "two sessions" and elections; when the virus spread to Beijing in early March, the national "two sessions" were taking place there, and the NPC, the State Council and the CPPCC were also busy with elections.
} 


\subsubsection{New Consensuses in Disaster Management Research Call for New Theory}

\subsubsection{Two Consensuses}

As disasters are increasing in frequency and intensity, disaster management research has attracted more attention and made new progress. The following two consensuses have been reached.

First, disasters are a result of interactions between nature and society. The Environment as Hazard, first published in 1978, for the first time extends the scope of disaster mitigation from natural hazards and protective works to human reactions to disasters, pointing out that the influence of and losses incurred by disasters can be reduced by adjusting human behavior. ${ }^{3}$

Second, the social attributes of disasters begin to predominate over their natural attributes. As Anthony Giddens put it, "In all traditional cultures, one could say, and in industrial society right up to the threshold of the present day, human beings worried about the risks coming from external nature-from bad harvests, floods, plagues or famines. At a certain point, however - very recently in historical terms-we started worrying less about what nature can do to us, and more about what we have done to nature. This marks the transition from the predominance of external risk to that of manufactured risk."4

The above consensuses show that disaster management, as an integrated system, has two important implications. The first is comprehensive management, meaning we should manage both the natural and social attributes of disasters; the second is whole-process management, that is, managing not only the hazard-inducing social environment but also the social consequences caused by disasters. This is because the social attributes of the disaster include the hazard-inducing social environment and also disaster mitigation and relief operations. The two aspects influence each other and together determine the social consequences of the disaster.

This undoubtedly poses great challenges to research in social sciences since natural sciences and engineering technology have long provided more of the discourse system and analytical tools for disaster research and management. Of course, Western social scientists have made their efforts in this area. In Europe where formulation of theory has been a traditional strength, and the theory of risk society, which was developed by Ulrich Beck, Anthony Giddens and Scott Lash, has gained influence as an explanation of the world's disasters. In the United States, Enrico Quarantelli established the world's first Disaster Research Center (DRC) in the field of social sciences at Ohio State University in 1963. The research center has so far carried out nearly six hundred field studies and obtained a wealth of research results useful for policymaking. Although China has suffered from frequent disasters since ancient

\footnotetext{
${ }^{3}$ Ian Burton, Robert W. Kates \& Gilbert F. White. The Environment as Hazard. New York: The Guilford Press, 1993.

${ }^{4}$ Anthony Giddens. Runaway World: How Globalization is Reshaping Our Lives (trans. Zhou Xiaohong). Nanchang: Jiangxi People's Publishing House, 2002, p. 23.
} 
times and has practical experiences in dealing with them, theoretical research on disasters is inadequate, especially in the field of social sciences. Therefore, it is essential to construct a new analytical framework to provide intellectual support for explaining and responding to disasters.

\subsubsection{Three Questions}

From a methodological point of view, whether a theoretical framework is well developed mainly depends on how well it may be used to explain the reality or to make predictions. A theoretical framework for disaster management should focus on addressing the following three questions.

First, social dimension. This means that the theoretical framework, based on the basic concepts and ideas of social sciences, should provide an explanation about the social attributes of disasters and be helpful to disaster management.

Second, integrated approach. The theoretical framework should be built on the basis of research findings in both social sciences and natural sciences. Only in this way can the framework produce public knowledge that faithfully reflects reality and meet the requirements of integrated disaster management.

Third, local relevance. The theoretical framework should ultimately provide valuable analytical tools for disaster management in China, and whether the tools are effective depends on how well they can be used to explain China's problems. This, however, does not mean rejecting international dialogues.

The existing conceptual system and theoretical paradigm in China's disaster research are not capable of addressing the three questions at the same time. Because of the classification of academic disciplines, disaster research in the field of social sciences lacks a holistic approach. Representative studies fall into three different fields and span three different periods. First, beginning in the early 1990s, sociologists paid attention to social risks in China's reform and opening up. Some focused on quantitative risk analysis and prediction, and some on diagnosis and criticism of structural transformation. Second, given the challenges faced by governments around the world after the September 11 attacks in 2001, researchers in political science showed growing interests in how the Chinese government conducted crisis management against the backdrop of governance reform in the transition period. Third, after the SARS outbreak in 2003, researchers and practitioners in administrative management, driven by national needs, carried out intensive and large-scale research on the institutions, mechanism and legal framework governing government crisis management. Research in these three fields has different focal points: sociological research, on the basis of observation of social phenomena, tries to predict trends through the index system and structural diagnosis; research in the political sphere, on the basis of international comparison, pays close attention to how policy makers could benefit from international good practices and how the political consequences of disasters would propel governance reform; and research in administrative management, driven by the practical needs, emphasizes the design and operation of 
general rules and principles and the immediate control of disasters. On the whole, research in the three fields focuses on one of the three different phases, rather than the whole process, of disasters and disaster management.

How then should we go about constructing an analytical framework for studying disaster management that does justice to the social dimension of disasters, brings together the social and the natural sciences, and is applicable to the Chinese context? This study tries to answer the following questions: first, how can we, drawing on both research findings in natural sciences and the concepts and theories of social sciences, establish a platform for communication across disciplines such as sociology, politics and administrative management? Second, how can the platform benefit integrated disaster management, facilitating comprehensive and whole-process analysis of disasters and the formulation of a corresponding management framework? Third, given the global challenges of disasters China is facing and its own pain of transition, how can we provide a well-substantiated explanation for this reality?

\subsection{Intellectual Genesis}

\subsubsection{Three Research Traditions}

Human beings have been concerned about disasters since ancient times. Over the past several millennia, people have been faced with all kinds of disasters, and never stopped fighting and studying them. Noah's Ark in The Old Testament of the Bible is one of earliest myths about flood. The Chinese civilization is generally believed to begin from the era when Yu the Great tamed the flood. During the long years of disaster research, three traditions have persisted.

\section{1. "Engineering-technology" tradition}

This is the oldest of the three research traditions, as the mankind's first collective experience of fear came from natural disasters. Before science could provide reasonable explanations, people developed a set of symbols and rituals such as offering sacrifices to protect themselves from disasters. With the advances in natural sciences, people got a better understanding of natural disasters. They tried to find out the causes of disasters, and took engineering and many other means to deal with them, such as embanking and weather forecasting. In the industrial era, explosion, gas leakage and other man-made disasters increased, so countermeasures such as fire extinguishers and gas masks were created. Most researchers who hold to this tradition work in the fields of natural sciences and engineering. They generally believe and are optimistic that disasters can be prevented and controlled through engineering, education and enforcement.

2. "Organization-system" tradition

During the Cold War, people realized that international conflicts may bring even greater harms than natural disasters and decision-makers, depending on the organi- 
zational or institutional settings, could have an impact on foreign relations. ${ }^{5}$ Humanity was facing the question of survival. A case in point is the Cuban Missile Crisis in 1962. After that, disasters such as the 1979 Three Miles Island accident in the United States, the 1984 Bhopal disaster in India, and the 1986 Space Shuttle Challenger explosion brought people's fear and concern from international conflicts to the everyday world. Underlying all these disasters are problematic organizational management and flawed institutional design. Researchers who uphold this tradition mostly have a research focus on management. Also optimistic about the future, they try to prevent, identify, isolate, tackle and manage disasters by strengthening organizational management and improving institutional design. ${ }^{6}$

\section{3. "Politics-society" tradition}

Since the 1980s, far-seeing researchers have come to realize that the greatest disaster is the self-destruction of modernity. As Ulrich Beck pointed out, in the process of modernization, the incredible productivity gains have given rise to unprecedented dangers and hazards. "The possibility of nuclear war, ecological calamity, uncontainable population explosion, the collapse of global economic exchange, and other potential global catastrophes provide an unnerving horizon of dangers for everyone". 8 Most of the researchers upholding the tradition work in social sciences, especially sociology and politics. They believe that the root of the problem lies in modernity per se-reflexive modernity, ${ }^{9}$ and the way out is political and social transformation. However, that is not going to be easy. It will be held back by the inertia of society and the people, and meet resistance from the vested interest. Therefore, researchers in this field are generally pessimistic.

\subsubsection{Three Core Concepts}

Each of the three research traditions has at its core a concept that defines its research perspective and approach.

\subsubsection{1 "Disaster" in the "Engineering-Technology" Tradition}

It is difficult to verify when "disaster", as a strictly defined concept, became a new subject of study. Nonetheless, it has been widely agreed over the years that: (1) there

\footnotetext{
${ }^{5}$ Charles F. Hermann. Crises in Foreign Policy: A Simulation Analysis. Indianapolis: Bobbs-Merrill, 1969, p. 14.

${ }^{6}$ Steven Fink. Crisis Management: Planning for the Inevitable. New York: Backinprint.com, 2000.

${ }^{7}$ Ulrich Beck. Risk Society (trans. He Bowen). Nanjing: Yilin Press, 2004, p. 15.

${ }^{8}$ Anthony Giddens. The Consequences of Modernity (trans. Tian He). Nanjing: Yilin Press, 2000, p. 125.

${ }^{9}$ Ulrich Beck, Anthony Giddens, and Scott Lash. Reflexive Modernization: Politics, Tradition and Aesthetics in the Modern Social Order (trans. Zhao Wenshu). Beijing: The Commercial Press, 2001.
} 
are both natural and man-made disasters; (2) natural disasters include floods, earthquakes, hurricanes (typhoons), droughts, mudslides, sandstorms, ice and snow, and cold waves, and man-made disasters include work-related accidents, environmental pollution, epidemics, toxic food, and so on; (3) the main consequences of disaster are casualties, property losses, and social disorder; (4) natural and man-made disasters both fall into three categories by severity: everyday emergency, community disaster, and catastrophe; and (5) the basic coping strategy is to understand the root causes of disasters, warn people about them, get prepared and respond to disasters through engineering and other technological means.

Among natural disasters, earthquakes and floods are most extensively researched. Through systematic research on the mechanical mechanism of the earth's lithosphere at the macro and micro levels, and analysis of numerous real cases, researchers have made it possible to give early warning of earthquakes and apply risk assessment results to architectural designs. Japan has been taking the lead worldwide in seismological research, seismic design of building structures, and earthquake engineering. Researchers in Europe and the United States excel in flood simulation and risk assessment, and they try to monitor and control floods by studying flood dynamics and flood forecasting models. In the field of man-made disasters, work-related accidents have been the most researched area.

\subsubsection{2 "Crisis" in the "Organization-System" Tradition}

American scholar Charles F. Hermann is generally acknowledged as the first researcher to provide a rigorous definition of "crisis" and make it a specialized area of study. He defined crisis as a situation that "(1) threatens high-priority values of the organization goals, (2) presents a restricted amount of time in which a decision can be made, and (3) is unexpected or unanticipated by the organization". ${ }^{10}$ This definition, which points out crisis management is essentially about decision making, had greatly influenced crisis research before the 1980s.

Graham Alison, based on the case study of the Cuban Missile Crisis, proposed three influential models of decision making in crises: (1) rational actor model; (2) organizational procedure model; and (3) governmental politics model. ${ }^{11}$

On the basis of the "bounded rationality" idea of Herbert Simon, Irving Janis studied the process and conditions of decision making from psychological and individual perspectives, and put forward three rules about decision making: (1) cognitive decision; (2) affiliative decision; and (3) self-serving and emotive decision. ${ }^{12}$

After the 1980s, while tensions between countries began to ease, tensions within a social system emerged. Against this backdrop, crisis was refined as a serious threat to the basic structure and core values of a social system, in which key decisions are

\footnotetext{
${ }^{10}$ Charles F. Hermann. Crises in Foreign Policy: A Simulation Analysis. Indianapolis: Bobbs-Merrill, 1969, p. 14.

${ }^{11}$ Graham Allison. Essence of Decision. Boston, Mass.: Little Brown \& Company, 1971.

${ }^{12}$ Irving L. Janis. Crucial Decision: Leadership in Policymaking and Crisis Management. New York: The Free Press, 1981.
} 
required because of the high uncertainty and time pressure (Uriel Rosenthal et al. 1989) ${ }^{13}$ Later, more researchers started to explore a broader range of crisis management issues, and paid attention to the whole process of crisis and crisis management. For example, Steven Fink divided the crisis life cycle into four stages: prodromal, breakout or acute, chronic, and resolution. Norman R. Augustine identified six stages of crisis management: avoid crises, prepare to manage crises, recognize a crisis, contain the crisis, resolve the crisis, and profit from the crisis. And Robert Heath proposed the $4 \mathrm{R}$ model: reduction, readiness, response, and recovery.

\subsubsection{3 "Risk" in the "Politics-Society" Tradition}

Originally, "risk" was a term in insurance, meaning the probability of losses. Frank Knight then defined it as an economic term by differentiating risk from uncertainty, and used uncertainty to explain where profit comes from and what the nature of businesses is. ${ }^{14}$

Apart from substantive definitions of "risk", sociologists, psychologists and anthropologists have also developed constructive definitions of the term. According to sociologist Nicklas Lumann, what's opposite to risk is not security but danger. The distinction between the two notions is that risk depends on how people perceive it, so does the risk associated with it, while danger precedes human decision or behavior, and the loss associated with it is determined by external factors. ${ }^{15}$ Psychologist Paul Slovic holds that risk is subjectively defined by individuals who are subject to influence by psychological, social, institutional and cultural factors. With well-designed surveys, these factors and their relationships can be measured through quantitative and modelling methods. ${ }^{16} \mathrm{He}$, together with Sarah Lichtenstein and Brauch Fischhoff, established a measurable model for risk perception. Risk is not only related to individual perception, argued anthropologist Mary Douglas and political scientist Aaron Wildavsky, but more importantly, should be seen as a "joint product of knowledge about the future and consent about the most desired prospects" ${ }^{17}$ On this basis, they divided risks into three categories: social and political risk, economic risk, and natural risk, and attributed the evolution of social fabric to three distinct cultural types: hierarchical culture that sees social and political risk as the greatest risk, market individualistic culture that views economic risk as the greatest, and marginal culture that regards natural risk as the greatest.

\footnotetext{
${ }^{13}$ Uriel Rosenthal, Charles Michael \& Paul Hart, eds. Coping with Crises: The Management of Disasters, Riots, and Terrorism. Springfield: Charles C Thomas Pub. Ltd., 1989, p. 10.

${ }^{14}$ Frank Knight. Risk, Uncertainty, and Profit (trans. An Jia). Beijing: The Commercial Press, 2007.

${ }^{15}$ Niklas Lumann. Risk: A Sociological Theory. Berlin: de Gruyter, 1993, p. 9.

${ }^{16}$ Paul Slovic. "Perceptions of Risk: Reflections on the Psychometric paradigm", in Sheldon Krimsky and Dominic Golding, eds., Social Theories of Risk. Westport: Greenwood Press, 1992, p. 120.

${ }^{17}$ Mary Douglas \& Aaron Wildavsky. Risk and Culture. Berkeley: University of California Press, 1982, p. 5.
} 
Ulrich Beck and Anthony Giddens made "risk" a general term. In their statements about risk society, risk, as a basic feature of society and what is inherent in modernity, fundamentally changes the operational rules, dynamics and basic structure of industrial society. Beck argues that from the historical perspective of social evolution, risk distribution will overlap with and ultimately merge with wealth distribution. ${ }^{18}$ Modern risks have shaken up the relationship between the present, the past and the future. It is no longer the past, but the risk of the future, that determines our choice today. ${ }^{19}$ While in some areas and lifestyles, modernity has reduced the overall risk, but it has also introduced some new risk parameters that people had known little or nothing about in the past, and these parameters involve high-consequences risks deriving from the globalization of modern social system. ${ }^{20}$ As Beck further pointed out, people may, for the first time, feel the sense of a kind of common destiny, because the threat is amplifying infinitely, the everyday consciousness of cosmopolitanism is awakened, and even the boundaries between mankind, animals and plants may be eliminated: whereas risk would contribute to the formation of risk society, global risk contributes to a global risk society. ${ }^{21}$

\subsubsection{Trend Towards Disaster-Centered Integration}

The research traditions represented by the three core concepts, though relatively independent of each other, show a trend towards integration. In 1945, Gilbert White published his influential dissertation titled "Human Adjustment to Floods", starting to study natural disasters from the angle of human behavior. In 1978, he co-authored The Environment as Hazard with Ian Burton and Robert Kates, which for the first time shifts the focus of disaster mitigation from hazards and engineering to human reactions to disasters, pointing out that adjusting human behaviors helps relieve the impacts of and losses from disasters. Such transition not only has great influence on disaster research in natural sciences, but also given rise to a surge in social science research on disaster. Enrico Quarantelli, an influential American disaster scholar and founder of the Disaster Research Center (DRC), concluded after reviewing disaster research in the West in the past four decades that (1) the classification system that distinguishes between natural and man-made disasters cannot explain the social attributes of disasters, and it is better to divide disasters into consensus type and dissensus type. Consensus-type disasters are those where there is an agreement on the meaning of the situation, the norms and values that are appropriate, and priorities that

\footnotetext{
${ }^{18}$ Ulrich Beck. Risk Society (trans. He Bowen). Nanjing: Yilin Press, 2004, p. 1.

${ }^{19}$ Anthony Giddens. Modernity and Self-identity: Self and Society in the Late Modern Age (trans. Zhao Xudong \& Fang Wen). Beijing: SDX Joint Publishing Company, 1998.

${ }^{20}$ Anthony Giddens. Modernity and Self-identity: Self and Society in the Late Modern Age (trans. Zhao Xudong \& Fang Wen). Beijing: SDX Joint Publishing Company, 1998. p. 4.

${ }^{21}$ Ulrich Beck. What is Globalization? (trans. Chang Hefang; eds. Xue Xiaoyuan). Shanghai: East China Normal University Press, 2008, p. 43.
} 
should be followed. The dissensus-type disaster means there are sharply contrasting views of the nature of the situation, what brought it about, and what should be done to resolve it. ${ }^{22}$ (2) Except in those extremely vulnerable areas, people generally pay little attention to disasters before they happen, whereas in regions frequently struck by disasters, relevant organizations and their activities will promote the formation of a disaster sub-culture. (3) Disasters usually cause visible psychological reactions such as insomnia, lack of appetite, and irritability, but these effects are non-clinical, selfhealing, and last for a short period. The victims rarely show functional inadaptability at home, school or work, and many of them could demonstrate similar syndromes even if the disaster had not happened. (4) Written emergency plans, though legally necessary, are by no means as important as emergency procedures. (5) The greater the disaster, the more likely new organizational structure and functions, hence greater difficulty in coordination. Hence, the key to catastrophe response is coordination. (6) The media play double roles, both as outsider and part of the emergency response system. These two roles are difficult to integrate. ${ }^{23}$

According to Quarantelli, the Western social science studies of disaster have been very fruitful, though there are also serious challenges. For example, as scholars oriented in different disciplines join the disaster research, it is even difficult for them to agree on the definition of disaster; the differences in their understanding of basic concepts and assumptions tend to cause confusion and inconsistency. Quarantelli holds that it is necessary to get a big picture of the research subject by creating a new paradigm for disaster research. In this new paradigm, disaster is a type of crisis scenario, and theoretical analysis of risk will to a great extent influence the orientation of the disaster research in social sciences. ${ }^{24}$ As for the trends of crisis and crisis management, Rosenthal thinks that future crisis will be essentially complex, a causal relationship full of uncertainty and a "self-perpetuating circle", and the key trends include transnationality, media, scientific and technological advances, and dissipation of government authority. ${ }^{25}$ These views echo the theory of risk society.

Over time, researchers have got a deeper insight into reality, and new traditions and concepts have emerged and started to dominate. New traditions and concepts extend the study in breadth and depth, rather than replace the three original traditions and the three core concepts. Still, we include the three concepts of disaster, crisis and risk into disaster research, and refer to the product of such infusion as the social science disaster research out of the following considerations: first, compared with crisis research and risk research, disaster research has a longer history and is more fruitful; second, disaster research, which used to fall into the scope of natural sci-

\footnotetext{
${ }^{22}$ Enrico L. Quarantelli \& Russell R. Dynes. Response to Social Crisis and Disaster. Annual Review of Sociology, Vol. 3, No. 2, 1977, pp. 23-49.

${ }^{23}$ Sue McNeil \& Enrico L. Quarantelli. Past, Present and Future: Building an Interdisciplinary Disaster Research Center on a Half-Century of Social Science Disaster Research, paper at international conference on Risk, Crisis and Public Management, Nanjing, 2008.

${ }^{24}$ Enrico L. Quarantelli. What Is a Disaster? Perspectives on the Question. London: Routledge, 1998, pp. 268-270.

${ }^{25}$ Uriel Rosenthal, Arjen Boin \& Louise K. Comfort, eds. Managing Crisis: Treat, Dilemmas, Opportunities. Springfield: Charles C. Thomas Pub. Ltd., 2001, pp. 11-14.
} 
ences and engineering, began to shift its focus to the social attributes of disaster and social governance, and social science scholars are encouraged to engage in crisis and risk research; third, an inherent link exists between the three core concepts of disaster, crisis and risk, with disaster (or more accurately, "emergency", a term closely associated with "disaster") at the core. Whereas "disaster" (emergency) refers to all sorts of natural and man-made events that cause great losses, "crisis" refers to the impacts these events have produced on the closely interconnected, ever-changing and complicated world and "risk" refers to the causes of the disaster and crisis. Currently, disaster research in social sciences still needs the underpinning of the three core concepts.

\subsection{Internal Logic ${ }^{26}$}

\subsubsection{From Disaster to Emergency}

In China, "disaster" is a term that draws widespread attention. In traditional Chinese culture, the idea of "interaction between heaven and man" has huge influence, and "disaster" is always connected with "heaven", and is believed to reflect the will of heaven.

The traditional concept of "disaster" is now facing challenges. First, though the classification of disaster into natural and man-made allows a wide array of events to be included, it is increasingly difficult for the tradition concept of disaster to cover some types of event such as mass incidents. In this regard, Robert Stallings proposed that disaster should be considered as not only a natural or technical risk, but also an exception to the society's operational norm. ${ }^{27}$ While Western countries can use the natural and man-made disaster classification to increase the scope of disaster, the Chinese term of "man-made misfortune" is inappropriate, and the term "natural calamity" is even more so. Second, it is necessary to think out of the traditional concept of "disaster" and to understand disasters and disaster response from a broader and longer-term perspective.

Given these considerations, a more appropriate concept should be "emergency", or "public emergency" which means the same in this book. In fact, "emergency" is not a strictly defined academic concept, but compared to "disaster", it has at least two advantages. First, it is highly abstract, comprising all kinds of incidents, whether they are a natural disaster, a human-induced accident, or even "an exception to the society's operational norm". Second, it is neutral. Disasters are no longer classified

\footnotetext{
${ }^{26}$ See: Tong Xing \& Zhang Haibo. An Analytical Framework of Disaster Management in the Context of China. Social Sciences in China, 2010 (1): 132-146.

${ }^{27}$ Robert A. Stallings. "Disaster and the Theory of Social Order", in Enrico L. Quarantelli, eds. What Is a Disaster? Perspectives on the Question. London: Routledge Press, p. 137.
} 
as external, man-made, and unusual. They are all sudden and unexpected. Given the current political and social context of China, the concept of "emergency" is more acceptable to people.

This change can be seen in both practice and institutional arrangements. China's disaster management has gone through several stages, from disaster relief, disaster prevention and mitigation, to comprehensive disaster reduction, and is moving towards the stage of emergency management. In the face of disasters, in addition to primitive rituals, the most natural reaction of mankind is disaster relief. Early measures included rescue, tax relief, relocation, transfer of grain, and compensation. Later, measures such as building water conservancy facilities, adjusting plans and storing grain were developed for the purpose of disaster prevention and mitigation. At the stage of comprehensive disaster reduction, man-made disasters, in addition to natural disasters, were also included in the scope of disaster prevention and mitigation. From the 1950s, disaster management in China focused on disaster relief and disaster prevention at the early stage. The government successively set up disaster management agencies such as the Ministry of Water Resources, the Ministry of Internal Affairs, the Central Disaster Relief Committee and the Ministry of Civil Affairs. After China joined the UN's program of the International Decade for Natural Disaster Reduction (IDNDR) in 1990, the concept of comprehensive disaster reduction gained popularity. After the close of the program, China International Disaster Reduction Commission established during the decade was renamed China National Commission for Disaster Reduction, and remains the country's top agency for natural disaster reduction. However, limited progress was made in comprehensive disaster reduction. For example, a majority of man-made disasters in China were work-related accidents, and the highest authority dealing with them was the State Administration of Work Safety, instead of the National Commission for Disaster Reduction. This situation did not change until the SARS outbreak in 2003. In response to the threats of new infectious diseases and frequent occurrence of other emergencies, the Chinese government decided to establish an emergency management system, at the core of which is a comprehensive strategy for emergency response that consists of plans, institutions, mechanisms and rules and regulations. In this system, "disaster" is replaced by the more abstract concept "emergency", which is broadly defined as "a sudden natural disaster, accident, public health incident or public security incident that has caused or may cause serious social consequences, and warrants immediate action", and response to such emergencies is referred to as "emergency management". Since then, at the institutional level, China has directly moved from the stage of disaster prevention and mitigation to comprehensive emergency management. 


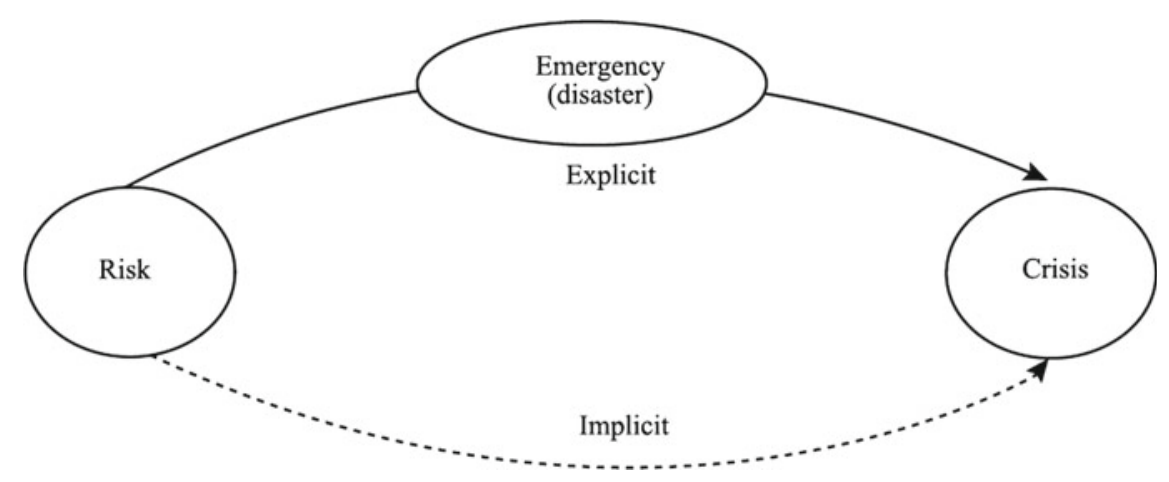

Fig. 1.2 Logical relationship between risk, emergency (disaster) and crisis

\subsubsection{The Continuum of "Risk-Disaster (Emergency)-Crisis"}

While the transition from "disaster" to "emergency" addresses the conceptual issue concerning "disaster", the subsequent problem is how to place "emergency" in the "closely interconnected, ever-changing and complicated world ", which requires a look at the relationship between emergency, risk and crisis. Evidently, the same is true of "risk" and "crisis". If risk prediction cannot be translated into management strategy, its significance will be quite limited; as to crisis, a "full-fledged theory" is needed to explain the causes and evolution of different types of crises and the pattern of crisis evolution so as to better understand crisis or crisis management. The relationship between risk, emergency (disaster) and crisis is shown in Fig. 1.2.

"Emergency" is a broader term than "disaster", but its logical relationship with risk and crisis remains unchanged. At the conceptual level, risk has become a general term, "marking the beginning of a new era"; crisis has become part of our everyday world, and an emergency is a public incident. This indicates that the three concepts are consistent in terms of publicness.

As to the logical relationship between risk and crisis, at the level of publicness, risk refers to a kind of uncertainty that can result in large-scale losses, and is, in essence, a possibility; crisis refers to the political and social consequences caused by some kind of loss, and is, in essence, a fact. Therefore, risk precedes crisis, and there is a causal relationship between the two; in other words, the fundamental cause of the consequences of crisis is risk. Thus, we can make the following inferences.

Inference 1: The nature of risk determines the nature of crisis.

The causal relationship between risk and crisis, however, is only potential. Such implicit causality only becomes explicit through emergency. Certainly, experts in specific fields are aware of such underlying causal relationship. Once "emergency", like a trigger, occurs, the explicit causal relationship between risk and crisis will 
become an iron-clad fact that the general public and even headstrong leaders have to admit. In reality, explicit causal relationship manifests itself in two kinds of situations.

(1) Sometimes one large-scale emergency can expose the underlying causal relationship between risks and crisis, which is suddenly cast under the spotlight, attracting the attention of many.

(2) A number of small-scale, little-noticed emergencies gradually expose the underlying causal relationship between risk and crisis, a slow process that is not easy to perceive.

The two different paths from risk to crisis correspond to the classification of crises in the West: instant crisis, and creeping crisis.

Based on the above causal chain, the following two inferences, which have important implications for emergency management practice, can be drawn.

Inference 2: The root cause of crisis is risk, not emergency.

Inference 3: The real consequence of risk is crisis, not emergency.

In the symmetric causal chain, emergency stands between risk and crisis. The distance between emergency and risk denotes the degree of risk accumulated, and a longer distance means a greater degree of risk; the distance between emergency and crisis can be interpreted as the severity of crisis, and a longer distance indicates that the crisis is less serious. Thus, another two inferences can be made.

Inference 4: The greater the accumulated risks, the more serious the crisis.

Inference 5: The less the accumulated risks, the less serious the crisis.

It goes without saying that risk and emergency cannot be separated. Risk can amplify the consequences of emergency. A small emergency, after risk amplification, can become a large-scale one. This leads to another inference.

Inference 6: The greater the accumulated risks, the more serious the consequences of emergency.

Since the consequences of emergency are subject to the influence of social risk, another inference can be made.

Inference 7: The nature of an emergency is decided by that of risk.

Under the premise that risk has been created systematically, emergency itself is a kind of risk mitigation, and serves as a "safety valve" or "buffer" as called in conflict theory in social sciences. This leads to the eighth inference.

Inference 8: Emergency is a bad thing, but can be a good one as well.

There is no denying that the SARS outbreak in 2003 was a disaster, but it upended China's obsession with GDP, and became one of the reasons the central government put forward the Scientific Outlook on Development in the autumn of that year. Since then, emergency management has practically become a source of management 


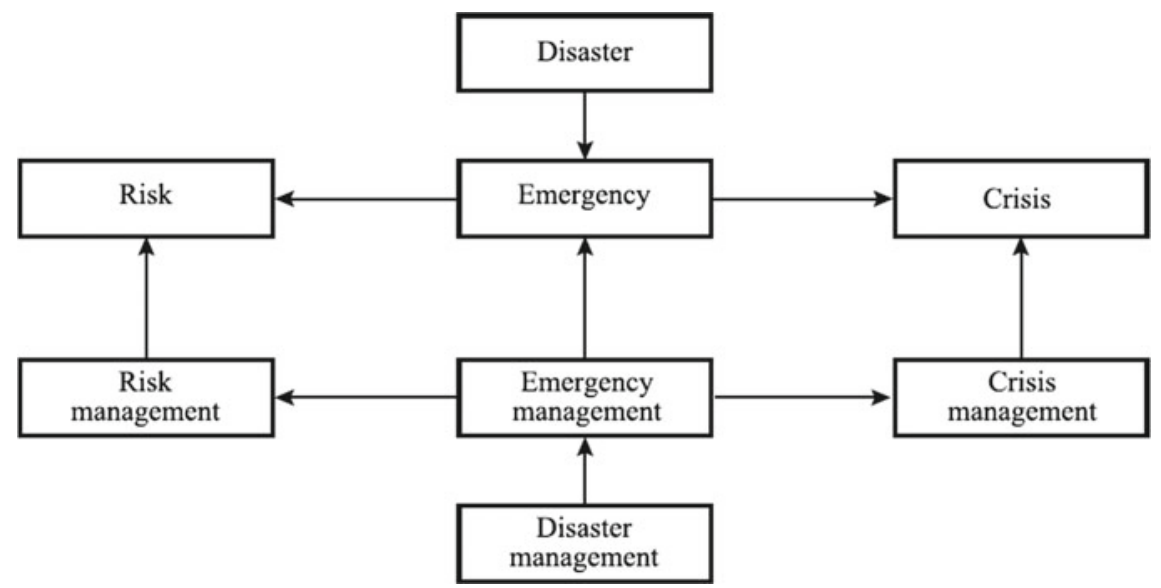

Fig. 1.3 Logical relationship between disaster management, emergency management, risk management, and crisis management

innovation in China. For example, the administrative accountability system began with the dismissal of then Minister of Health Zhang Wenkang and Mayor of Beijing Meng Xuenong, who were held accountable for ineffective response to the SARS outbreak. After that, it is common that officials are held accountable in the wake of emergencies. During the SARS outbreak, local authorities were required to report the number of confirmed cases, suspected cases, recovered patients and deaths and other data every day, and this promoted the establishment of the government information disclosure system. In addition, the approach of combining the institutions, mechanisms and rules and regulations to address major issues also originated from emergency management.

\subsubsection{Whole-Process Response System: “Risk Management-Disaster (Emergency) Management-Crisis Management”}

Judging from management practices, the original "disaster management" should be extended to "emergency management", which comes after risk management and precedes crisis management. Their relationship is illustrated in Fig. 1.3.

While the scope of emergency management is limited to the emergency situation per se, the so-called whole-process response system represents a holistic approach to the problem. According to Inference 2, emergency management cannot effectively reduce the crises the government has to deal with. The most fundamental problem China is facing is risk, rather than emergency. Only the whole-process response system integrating risk management, emergency management and crisis management 
can help eliminate risk and shake off crisis. In view of this, we may refer to the "wholeprocess response system" as "emergency management" in the broader sense, and have it incorporated into the national framework for the optimization of governance structure. The integrated risk, emergency and crisis management system should have the following features.

First, systematic management. Risk management, emergency management and crisis management are all an indispensable part of the system, and they work together to keep the events under control, reduce losses, restore government image, strengthen political legitimacy, promote social reform, improve governance structure and mitigate risks, so as to ensure long-term social stability.

Second, dynamic management. Ideally, corrective action needs to be taken before any risk evolves into a real emergency. In reality, however, such action may easily run into difficulty because of resistance and the lack of motivation. Therefore, emergency management can be taken as opportunity to promote crisis management, and then to propel risk management and crisis management.

Third, proactive management. Proactive management should take priority. Emergencies, after all, can cause casualties, loss of property, social disorder and other consequences, and there is no guarantee that every emergency can be kept under control.

To achieve integrated risk, emergency (disaster) and crisis management, there are three options or paths: first, focus on emergency (disaster) management, and further improve the emergency (disaster) management system; second, proceed from emergency (disaster) management to crisis management, and propel policy adjustment and reform; and third, advance risk management, and improve social fabric to fundamentally reduce uncertainty.

Considering the four types of emergencies in China, the paths can be selected in line with the following principles: for natural disasters and public health incidents, the first path is advised, with the other two playing a complementary role; for accidents, the second path should be given priority; and for public safety incidents, the third path should take the lead.

In practice, a three-step approach can be taken to achieve integrated risk, emergency and crisis management: in the near future, take path one by improving emergency (disaster) management and the system as a whole; in the medium term, take path two, advancing crisis management and promote policy adjustment and reform; in the long term, take path three, advancing risk management and optimizing social fabric to reduce uncertainty. 


\subsection{A Basis for Integrated Research}

\subsubsection{Risk Warning in Sociology and Crisis Management in Public Administration}

Based on the comprehensive analytical framework proposed above, the research on risk warning in sociology and that on crisis management in public administration can be integrated in the following ways.

\subsubsection{Conceptual Integration}

Theoretical research starts with defining concepts. Even for the same research subject, different disciplines may use different concepts due to different disciplinary backgrounds and research traditions.

\section{(1) Risk}

From an interdisciplinary perspective, Ortwin Renn sums up seven approaches to the conception and assessment of risk: the actuarial approach, the toxicological and epidemiological approach, the engineering approach, the economic approach, the psychological approach, social theories of risk, and cultural theory of risk. ${ }^{28}$ The seven approaches can be roughly divided into two categories: the first is technical orientation, which regards risk as a probability and usually uses the statistical method of expected frequency, such as the life table in insurance; the other is economicsocial-cultural orientation, which sees risk as a social consequence and usually uses the formula " $\mathrm{R}$ (risk) $=\mathrm{P}$ (probability) $\times \mathrm{H}$ (hazard)". These are also two divergent definitions of risk the UN recommends for the assessment of chemical toxicity. ${ }^{29}$

\section{(2) Social risk}

Frank Knight holds that a random state that can be expressed in probability is risk, while one that cannot be expressed (or at least under current conditions) is uncertainty. This dichotomy lays the foundation for the modern insurance industry to calculate risks, and also implies the nature of social risk. ${ }^{30}$ Social risk is usually incalculable and cannot be expressed in probability and in essence, is a kind of uncertainty.

\footnotetext{
${ }^{28}$ Ortwin Renn. "Concepts of Risk: A Classification". Social Theories of Risk. Greenwood Press, 1992 , p. 56.

${ }^{29}$ Mary Douglas. Risk Acceptability According to the Social Science. London: Routledge \&Kegan Paul, 1986, p. 20.

${ }^{30}$ Risk that can be expressed in probability is also called "calculable risk", and risk that cannot be expressed in probability is called "incalculable risk". This classification of risk has important practical significance, and gives rise to two different ways of handling risk. "Calculable risk" is usually handled by insurance companies, which conducts "centralized management" of scattered risk. A typical example is compulsory traffic insurance. For "incalculable risk", insurance companies refuse to provide protection with the excuse of "force majeure", so the government has to take over by conducting "decentralized management" of concentrated risk. A case in point is
} 


\section{(3) Crisis}

People often interpret the concept of "crisis" from two perspectives: "process", and "event". The former tends to regard "crisis" as a non-routine, or "crisis", while the latter tends to see "crisis" as an "emergency" or a series of "emergencies". Scholars usually take the "process" perspective and use the term "crisis", while policymakers generally take the "event" perspective and use "emergency". There are substantial differences between the two perspectives: the "process" perspective describes crisis as a continuous curve with "peak, "valley" and a complete life cycle, implying the possibility to prevent, control and warn against crisis; the "event" perspective regards crisis as a "point" and identifies whether or not a crisis will occur based on the specific state of events, and because this is simple in terms of operation, it is often adopted by government departments.

\section{(4) Public crisis}

Currently, Uriel Rosenthal's definition of crisis has been universally recognized in Chinese academia. According to him, crisis is a serious threat to the basic structure and core values of a social system, in which key decisions are required because of the high uncertainty and time pressure. Crisis is a general term, and public crisis is a particular form of crisis. At present, most researchers do not distinguish between "crisis" and "public crisis", but with time, the two should be separated. The former mainly appears in the study of corporate governance and management studies, while the latter gets a foothold in discussions about public governance and public administration.

From the above analysis, it can be seen that risk and crisis are different from each other and also connected. Risk refers to the possibility of loss, and crisis is the loss incurred; if without intervention, risk will eventually evolve into crisis. In other words, there is a causal relationship between risk and crisis; risk is the early form, crisis is the subsequent manifestation, and between risk and crisis is a "continuum". At the social level, risk logically turns into social risk and crisis into public crisis. Therefore, there is a causal relationship between social risk and public crisis, and between the two is also a "continuum".

(5) Early warning and emergency management

While studies of risk, social risk, crisis, and public crisis focus on risk and crisis per se, early warning and emergency management studies focus on the management approaches. Early warning is considered as the main approach to managing social risk, in the same way as emergency management to managing public crisis. The

the Wenchuan earthquake. This "incalculable risk" struck areas from Wenchuan to Qingchuan, and evolved into a public crisis. Relying on local governments and local resources alone was not enough to address the severe consequences, so the CPC Central Committee and the State Council designated 19 provinces (municipalities) including Guangdong, Shandong and Jiangsu to provide targeted assistance for post-disaster recovery and reconstruction of 17 seriously affected counties (cities) such as Wenchuan, Beichuan and Mianzhu. 


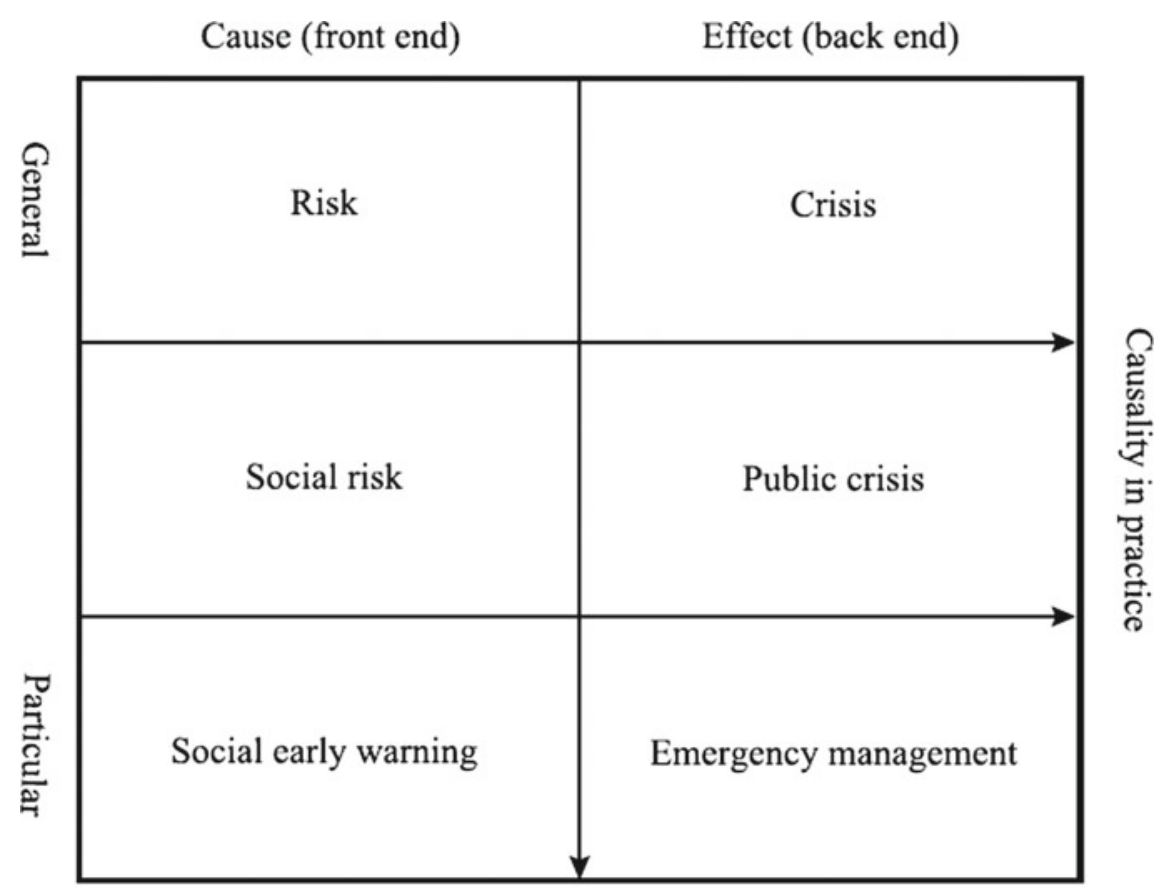

\section{Logical deduction}

Fig. 1.4 "Social risk-public crisis" integrated conceptual framework

process from social risk to early warning and from public crisis to emergency management also conforms to logical inference. The logical relationship between the six core concepts and their causal relationships in practice are shown in Fig. 1.4.

As can be seen from Fig. 1.4, social risk and public crisis are, respectively, the front end and back end of the same study. Social risk research and public crisis research are particular forms of the general risk research and crisis research, and early warning research and emergency management research are particular forms of social risk research and public crisis research. Traditional social risk and early warning research concentrated in sociology only involves the front end, while public crisis and emergency management research mainly in the sphere of public administration only involves the back end. Logical inevitability and practical causality both require the combination of social risk and early warning research with public crisis and emergency management research. The main topic of front-end research is how to identify, warn against and reduce social risk, but not all social risks can be accurately identified, timely warned against, or effectively reduced, and a considerable part of them will still evolve into public crises. In this case, the research in sociology can be of little help. In contrast, emergency management research in public administration only involves the back end. However, at the core of crisis management is the principle of "prevention outweighs control", and public administration research rarely addresses 


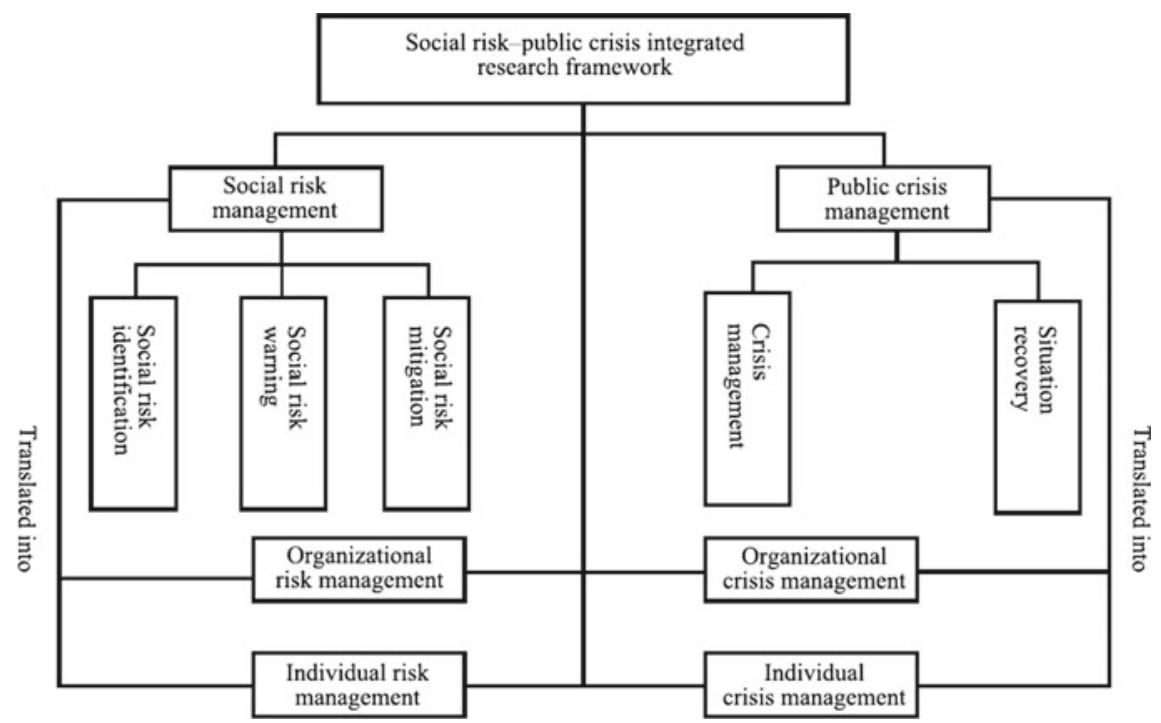

Fig. 1.5 "Social risk-public crisis" integrated research framework

social risk. Therefore, equal emphasis should be placed on the two: public crisis management must start from social risk management, and social risk management should be followed by public crisis management.

\subsubsection{Theoretical Integration}

Due to the practical causality between risk and crisis and their mutual transformation at individual, organizational and societal/public levels, the process of risk and crisis management should include at least two stages and three levels (Fig. 1.5). The two stages are the stage of social risk management and of public crisis management. The three levels include individual risk and individual crisis management, organizational risk and organizational crisis management, and social risk and public crisis management.

Social risk management includes three stages: risk identification, early warning, and risk mitigation. Risk identification is to identify and confirm social risks that pose threat to the normal operation of society, and grade them according to the degree of danger; early warning is to establish an early warning mechanism to grasp the accumulation of social risk and risk tolerance of society in a timely manner, and is the core link of social risk management; risk mitigation is to release and channel social risk close to the risk tolerance of society. Public crisis management includes two stages: emergency management and recovery. Emergency management is to make decisions in response to public crisis evolved from social risk, and allocate resources to minimize casualties and other losses, and it is the core link of public cri- 
sis management; recovery refers to the diagnosis and restoration of areas affected by public crisis, including the reconstruction of infrastructure, reconstruction of management mechanisms, restoration of social order, and psychological interventions. It is obvious that social risk and public crisis management research should be an integrated study that cuts across many disciplines, such as economics, management, psychology, sociology and public administration.

Throughout the process of social risk and public crisis management, the most important is early warning about risks and response to public crisis. Prevention takes precedence over control, so early warning is a top priority. Nonetheless, social risk in essence is uncertainty, and early warning is never omnipotent. Therefore, once social risk turns into public crisis, emergency management procedures should be initiated immediately.

Research on social risk and public crisis management is mainly concentrated in the fields of sociology and public administration. ${ }^{31}$ Some influential theoretical studies including those on social risk, risk society, anomie, social transformation and social control are essentially sociological research with a focus on description and explanation. Other important studies such as emergency management, crisis management and governance basically focus on countermeasures and applications from the perspective of public administration. Among them, social risk research and social control theory are interdisciplinary and address both social risk at the front end and public crisis at the back end. Some domains of their discourse fall into the scope of public administration, and others the scope of sociology. Though no important public crisis theories have been developed, researchers may draw on the above theories in order to achieve theoretical innovation (Fig. 1.6).

\subsubsection{Methodological Integration}

Different research methods of various disciplines can be integrated in a risk/crisis management study.

\section{(1) Index method}

In the 1960s and 1970s, because of American sociologist Romand Bauer's book, Social Indicators, the use of indicators gained ground in early warning research. In 1972, the Club of Rome published The Limits to Growth, a research report that gives a warning of the development model of mankind and has been regarded as a classic in the study of early warning indicators for social risk. Since China adopted the reform and opening up policy in 1978, Chinese scholars have started to use indicators to

\footnotetext{
${ }^{31}$ This is not always true. Risk research involves a wide range of fields such as actuarial science, economics, environmental science, toxicology, and epidemiology. Crisis research involves business management, journalism and communication, and public relations. The relationship between risk, social risk, crisis and public crisis as discussed earlier shows that it is difficult to separate these fields absolutely. However, it is justifiable to say that the existing theoretical achievements in social risk and public crisis research are concentrated in the two disciplines of sociology and public administration.
} 


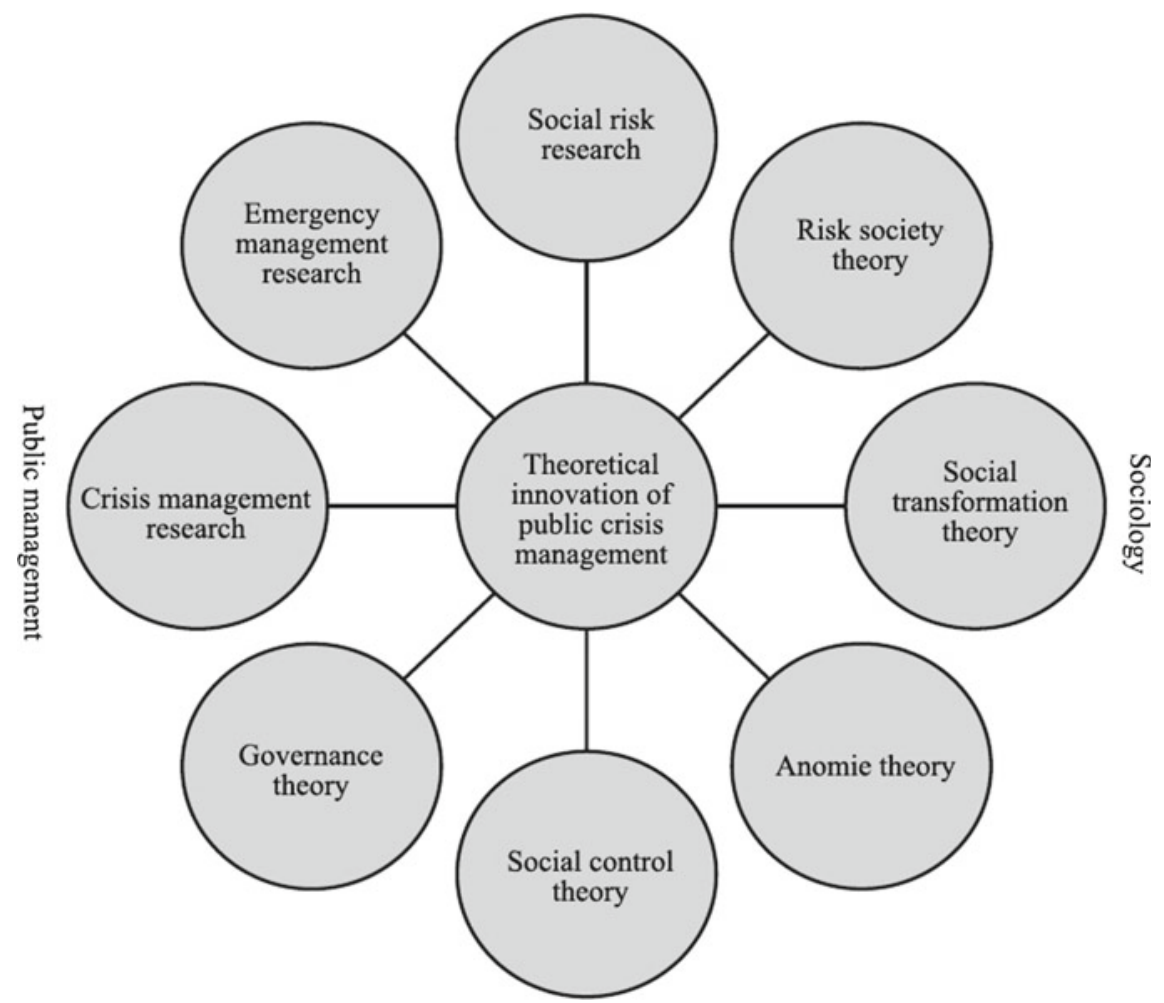

Fig. 1.6 Theoretical synthesis and innovation of public crisis management

study social risk warning. The index method is straightforward and operable, and thus liable to draw attention from policymakers. This research approach, however, faces two major challenges: one is the lack of convincing evidence in the selection of indicators and the assignment of weights, and the other is that it has not been validated by empirical facts. The basic presupposition of the index method is that risk is objective and quantifiable. In practice, researchers usually use the Delphi method to select indicators and assign weights, which in effect views risk as subjective, and this is logically conflicting. Moreover, the social system is too complicated to be compared to the computational system. A seemingly independent event in the social sphere may eventually lead to a big mess, while the comprehensive index system may not be ready to respond at all. In order for the index method to play a greater role in the study of public crisis management, new methods must be developed to meet these challenges.

\section{(2) Psychometric paradigm}

The psychometric paradigm is based on the assumption that risk is inherently subjective and is constructed by society. Since risk is subjectively defined by individuals 
who may be influenced by a wide array of psychological, social, institutional, and cultural factors, many of these factors and their interrelationships, with appropriate design of survey instruments, can be quantified and modeled. Through quantitative research, the psychometric paradigm reveals three basic dimensions of public risk perception: fear of risk, unknown risk, and exposure to risk.

These risks can be described with the following opposing variables: observable-not observable, known to exposed-not known to exposed, effect delayed-effect immediate, new-old, known to science-not known to science, controllable-uncontrollable, dread-not dread, global catastrophic-not global catastrophic, certain not fatal-certainly fatal, equitable-not equitable, individual-catastrophic, low risk to future generations-high risk to future generations, easily reduced-not easily reduced, risk decreasing-risk increasing, and voluntary-involuntary. Research finds that risk perception and attitude of the general public is closely related to the above variables. ${ }^{32}$

(3) Analogy

By analogy with combustion, social combustion theory points out that the process in which a social system moves from order to disorder and eventually a major public crisis occurs is essentially a process from quantitative change to qualitative change. ${ }^{33}$ Social combustion theory classifies the factors of social instability into external disturbances and internal contradictions, which, under the dual accumulation of quantity and quality and ignited by the "social trigger threshold", evolve into issues in focus. After sudden changes and fluctuations, two kinds of results will be produced: the original system will either collapse or hold on. In empirical research on social amplification of risk, some researchers applied the electronic model of 128 crises to database analysis, and to in-depth case study of risk-related community experiences. These analogies have been widely used in social risk research, but also aroused controversy and criticism. Analogy can stimulate researchers' imagination, but is difficult to reveal the inherent nature of the object of study.

\section{(4) Case study}

Since public crisis is manifested as a series of events, case study of the whole process of events is obviously an important method of public crisis research. However, the fact that social phenomena cannot be replicated makes it impossible to reconstruct the crisis. Although many researchers use case study as a research method to account for the process of public crisis management, it is of limited help to predict social risk and improve public crisis management.

\footnotetext{
${ }^{32}$ Paul Slovic. "Perception of Risk Reflections on the Psychometric Paradigm". Social Theories of Risk. Greenwood Publishing Group, 1992.

${ }^{33}$ Niu Wenyuan. The Social Physics and the Warning System of China's Social Stability. Bulletin of the Chinese Academy of Sciences, 2001 (1).
} 


\subsubsection{Integration of Topics}

An interdisciplinary research platform extending from social risk to public crisis should be set up. Currently, most studies on public crisis management only discuss how the government should respond to public crisis at the national level, but seldom dive into the mechanism of public crisis, especially the portents at the social level. Research on the "continuum" from social risk to public crisis can get a grip on the occurrence and evolution of public crisis through the process, and is therefore conducive to interdisciplinary collaboration and theoretical innovation. For policymakers, such research is clearly more effective and useful than simply considering issues from the perspective of public administration.

The key to the "continuum" research lies in how to establish an integrated response mechanism which requires an interdisciplinary research platform integrating early warning and emergency management. The response mechanism should first be based on risk classification, that is, targeted risk management should be conducted according to the types of risk; second, the mechanism should be based on risk grading, that is, emergency management should be geared to the different levels of early warning; third, it should also be based on crisis stages, that is, crisis management should vary depending on the different stages of crisis. The classified, tiered and phased risk and crisis response system should at least consist of the early warning mechanism, information mechanism, command mechanism, and collaboration mechanism.

Prevention outweighs control, so it is necessary to strengthen front-end research on public crisis. With the proposition of concepts such as "sustainable development", "Scientific Outlook on Development", "harmonious society" and "inclusive growth", a risk culture is taking shape in China in the process of social transformation. Now that social risks have been brought about by institutional factors, the focus should now be on how to understand, choose and avoid these risks. Changes in concepts can in turn mitigate objective social risks. The central government and local governments are now transforming the development model that places disproportionate emphasis on GDP growth, and paying more attention to social justice, wealth gap and environmental quality, and the government and official performance evaluation indicators are also changing.

Attention should be given to the creation of case study database and recovery from a public crisis. Often, high-tech on-site command equipment is wrongly believed to be what the public crisis emergency response system is all about. In fact, the more important part of the public crisis emergency management system is "software", that is, targeted emergency plans and emergency response drills. For local governments, an urgent task is to establish a case database for public crisis management, which, through the analysis of the emergency handling process of similar public crises, can provide empirical guidance for on-site decision-making. In addition, post-crisis recovery is also important, especially psychological recovery, which is now a blind spot in public crisis management at the local level. For example, after the gas well blowout accident of China National Petroleum Corp. in Kaixian 
County of Chongqing in 2003, smooth progress was made in emergency response and post-accident compensation; however, due to the neglect of social and psychological recovery, the local public morals declined dramatically after the disaster, and many people could not find the right direction of life for a long time.

\subsubsection{Integration of Research Resources}

In order to strengthen research on social risk and public crisis management, and achieve the integration of concepts, theories, methods and topics as mentioned above, research resources also need to be pooled together. At the national level, a common platform for early warning research in sociology and emergency management research in public administration should be established to promote an interdisciplinary approach that fosters exchange, involvement and integration. The teams of sociology, political science and management of the National Social Science Fund of China have taken initial steps in this regard by organizing cross-cutting research projects, providing mutual support for fund winners, and encouraging research teams to engage researchers from all relevant disciplines. The Department of Management Sciences of the National Natural Science Foundation of China has also started to provide funds for scholars conducting research in sociology and political science. At the micro level, research institutes, if conditions permit, can set up research centers for social risk and public crisis management, organize scholars from sociology, public administration, economics, psychology, environmental science and other relevant disciplines to carry out research projects together, which are preferably headed by those with both sociology and public administration (including administrative management) backgrounds.

\subsubsection{Integration of Research Ideas and Contents ${ }^{34}$}

A theoretical framework is the "skeleton" that supports the research ideas and contents of a social science study of disaster. The three core concepts of disaster, crisis and risk are interrelated. Crisis is the consequence of disaster; risk is the fundamental cause of disaster and crisis; risk and crisis are basically different stages of the same phenomenon and have a causal relationship: the former is the cause, and the latter is the result. Certainly, this kind of causality is implicit unless it is exposed by the occurrence of disaster. In view of the whole research process and in order of precedence, the social science study of disaster can be divided into three parts: risk research, disaster research and crisis research.

\footnotetext{
${ }^{34}$ See: Tong Xing \& Zhang Haibo. Social Science Disaster Research: Possibility of Interdisciplinary Integration. China Emergency Management, 2009 (3).
} 


\subsubsection{Risk Research}

On the one hand, risk is objective. The insurance industry, from which the term originates, and the risk society theory of Ulrich Beck and Anthony Giddens, among others, all hold that risk is an objective existence, no imagination. What is different is that risk, as an insurance term, refers to the possibility of losses individuals may suffer, while risk in risk society theory refers to the uncertainty that people in a specific area of the world may suffer losses.

On the other hand, risk is also subjective. Objective risk and subjectively perceived risk are usually not consistent. As Francis Ewald said, anything is not a risk in itself, and the world has no risk, but anything can be a risk, depending on how people analyze the dangers and consider the events. ${ }^{35}$ In other words, risk exists objectively, but the degree to which they are perceived by people is subject to social construction, which directly determines how people deal with risks. Therefore, risk research should answer the following four questions: (1) What particular risks exist in a specific area of the world? (2) How are these particular risks perceived by people, and what are the different perceptions? (3) How do these different perceptions turn into human effort to reduce risk? (4) To what extent can human effort reduce risk authentically? In a word, risk research includes four main aspects: risk identification, risk perception, risk reduction, and risk reduction assessment.

When trying to answer these questions, China's disaster research in social sciences should consider not only the geographical location but also the period of transition China is now in. At present, the country is facing both the "uncertainty of modernity" in the sense of global risk society and the "uncertainty of modernization" specific to a transitional country. ${ }^{36}$ Modernization implies basically the same thing as national transition. China's transformation has brought about significant changes in the four dimensions of economy, politics, society and culture. The old system centered on control and planning has gradually been shaken up, while the system centered on regulation and market has not yet been fully established. On the whole, a country's transformation is characterized by growing uncertainty, which largely accounts for social risk in the transition period. Corresponding to the four dimensions of national transformation, this uncertainty mainly takes four forms: the "by-product" of industrialization, regulatory loopholes, intense relationships, and value ambiguity. Their interactions with incidental factors have resulted in major disasters in China: environmental pollution, mining accidents, mass conflicts, and unsafe food.

\footnotetext{
${ }^{35}$ Francis Ewald. "Insurance and Risk", in Graham Burchell, Colin Gordon \& Peter Miller, eds. The Foucault Effect: Studies in Governmentality. Hemel Hemstead: Harvester Wheatsheaf, 1991. Cited from: Yang Xuedong. Risk Society and Order Reconstruction. Beijing: Social Sciences Academic Press, 2006, p. 12.

${ }^{36}$ It is generally acknowledged that modernization is the process to achieve modernity, that is, modernity is the goal of modernization, and modernization is the process toward modernity.
} 


\subsubsection{Disaster Research}

Disaster research concentrates on how to reduce the loss caused by disaster through adjusting human behavior, which is usually divided into four stages: mitigation, preparedness, response, and recovery.

(1) Disaster mitigation

Risk identification is the prerequisite of disaster mitigation, meaning disaster mitigation is only possible when risks are perceived at an early stage. This is where risk research and disaster research meet. Mitigation is not necessarily to "eliminate risk", but to reduce loss to an acceptable level. Even in the United States, mitigation is still one of the weakest links in disaster management practices. There are mainly two reasons. First, mitigation measures are generally proposed by academics rather than policymakers, and it is not easy to turn research issues into policy issues; second, mitigation measures need to be implemented down to earth, but general management departments lack the motivation to do so, because it is disaster management departments, not them, that will be responsible for the consequences of inadequate mitigation. Thus mitigation is not merely a technological issue. Only by reflecting on the negative consequences of social action from time to time and internalizing them can individual behavior and habits, organizational social responsibility and government policy preferences be developed, and a risk culture be fostered. Therefore, how to cultivate a risk culture is an important issue that derives from mitigation.

\section{(2) Disaster preparedness}

Emergency preparedness has been institutionalized in most countries, but two issues still need to be addressed: (1) From a qualitative perspective, what emergency management structure can achieve optimal efficiency and minimize casualties, property losses and social disorder in the wake of disasters? (2) From a quantitative perspective, what extent of disaster preparedness is sufficient to meet the needs of emergency management, without causing any waste of resources? The quantity of emergency resources should be "cost-effective" and also depends on emergency management structure. On the one hand, the cost of emergency preparedness is definitely lower than the consequences of disasters, since governments at all levels need to provide funds for many undertakings apart from emergency management. On the other hand, emergency management structure featuring regional cooperation can enable the sharing of resources, and to some extent help increase emergency resources available and reduce costs.

\section{(3) Disaster response}

The core of disaster response is coordination. Past experience suggests that the greater the disaster, the more likely there will emerge new behavioral structures and functions at the crisis time. A fourfold typology captures the phenomena well. Type I organizations are established ones that do not markedly change their general structure and functions at times of crises (e.g. many police and fire departments maintain 
their traditional forms and spheres of activity). Type II organizations are expanding ones that have new structures but old functions, such as the Red Cross. Type III organizations are extending ones that have old structures but new functions (e.g. a construction company using its traditional group structure to undertake building or street debris clearance). Finally, Type IV groups are new entities that carry out new disaster functions, such as informal search and rescue teams, or damage assessment groups. It appears that the greater the disaster, the more likely there is an increasing involvement of the organized entities going from Type I through Type IV. An ordinary, everyday emergency could be handled only by Type I organizations, but a catastrophic disaster will require the presence of all four types of organized behavior. It goes without saying that the presence of many such groups of differing structures and functions creates major problems of coordination at the community level. ${ }^{37}$ Just like the long-standing disputes over regional cooperation and hierarchical response in the above-mentioned government emergency structure, opinions are divided over which coordination mode is better: command-control, or cooperation. While the United States, where the cooperation mode dominates, has seen signs of the rise of the "command-control" mode, China should, in addition to sticking to the government-led "command-control" mode, make better use of the cooperation mode characterized by timely and flexible response. The cooperation mode can be adopted not only in dealing with everyday emergencies and community disasters but also in responding to catastrophes, as government agencies themselves may be paralyzed after the occurrence of a catastrophe. For example, after a devastating earthquake hit Wenchuan of Sichuan Province on May 12, 2008, a large number of non-governmental organizations, public interest organizations, and volunteers worked with government departments in disaster relief, playing an irreplaceable role in early disaster response.

\section{(4) Disaster recovery}

The key to disaster recovery lies not only in physical reconstruction and psychological recovery, but also in the formation of a learning mechanism, so that mitigation, preparedness, response and recovery can form a circular rather than a linear process. Therefore, it is necessary to assess the performance of disaster mitigation, preparedness and response, and include it into the government performance appraisal system.

\subsubsection{Crisis Research}

Crisis results from the interaction between disaster and the four factors of globalization, network, media and politicization, so a subjective perspective is more important than an objective perspective in crisis research. Emphasizing the constructive nature of crisis, Ajren Boin put forward a "political-symbolic" interpretative framework,

\footnotetext{
${ }^{37}$ Sue McNeil \& Henry Quarantelli. "Past, Present and Future: Building an Interdisciplinary Disaster Research Center on a Half-Century of Social Science Disaster Research", http://dspace.udel.Edu: 8080/dspace/handle/19716/3561.
} 
thinking that this framework can explain why people see certain events as crises, but at the same time rest easy with other events that look more threatening. ${ }^{38}$ This is also the main dividing line between crisis research and disaster research. Generally, disasters that are badly managed are most likely to evolve into crises, and "being badly managed" indicates that they are artificially constructed.

The network on which the dissemination and spread of crisis depends is the structural factor that determines the constructive attribute of crisis. The main nodes of the network include the general public, the media, business organizations, governments, non-governmental organizations, and intergovernmental organizations. In this network, the media play a great role, and to some extent, define crises. ${ }^{39}$ The media determine how people view crisis, and this ultimately affects the substantive attribute of crisis. In 2007, China promulgated the Emergency Response Law, which provides a legal basis for the news media to get engaged in emergency management, explore the policy, system and structural factors and values behind an incident, and propel reform accordingly. When the draft of the law was submitted to the National People's Congress for second deliberation, a widely controversial provision was deleted, which states that "in the event that the news media publish without authorization information on the handling of emergencies and state of incidents or report false information, and if the circumstances are serious or serious consequences are caused, they shall be imposed a penalty of RMB50,000 to RMB100,000 by the local government" ${ }^{40}$ This implies that the legislation encourages, rather than limits, the role of the media in emergency management.

The emergence and rise of the Internet has strengthened this logic. The Internet features anonymity and interactivity that the traditional media cannot match. The Internet allows users to express their views on policy issues more freely. Different from the traditional media, on the Internet, everyone can publish information, and information is disseminated in a multi-directional way and can spread to every corner of the world in an instant. These characteristics make it more difficult to control information, and as ordinary Internet users have a bigger say than ever before, the definition of crisis is no longer a matter that rests with just a few.

There is a logical relationship between risk research, disaster research and crisis research: risk identification serves disaster response, disaster response drives crisis dissemination, and crisis dissemination in turn propels risk identification, thus forming a cycle. In this case, disaster is not merely "misfortune", but shows a positive side: it can dynamically and continuously promote better performance of emergency management, so it follows that people no longer impracticably attempt to "prepare" for all risks.

\footnotetext{
${ }^{38}$ Arjen Boin. "Lessons from Crisis Research". In Managing Crises in the Twenty-First Century. International Studies Review, 2004 (6).

${ }^{39}$ Uriel Rosenthal. "21st Century Mega-Crises: New Challenges to Crisis Management”. Conference Paper, 2008.

${ }^{40}$ See: The 10th NPC Standing Committee Conducts Second Deliberation on Draft Law on Emergency Response. China Emergency Management, 2009 (6).
} 


\subsubsection{Integration of Research Methods and Research Techniques}

To answer these questions and construct the knowledge system of the social science study of disaster, corresponding research methods and techniques are required

\subsubsection{Methods and Techniques Specific to Certain Areas and Problems}

As risks are divided into natural risks and those induced by human activities, forecasts were first made about natural risks, and great progress has been made in the forecasts of meteorological disasters which can be very accurate today. Another catastrophic risk is earthquake, the forecast of which still remains a worldwide challenge today.

Out of risks of man-made disasters, the identification and prediction of visible hazards is relatively mature, as exemplified by risk prediction in workplace safety and work environment. In the early 1980s, China began to attach importance to the evaluation and control of major hazards, and in 2000, promulgated the National Standard for Identification of Major Hazard Installations (GB18218-2000). With regard to environmental risk monitoring, water and air pollution monitoring has become a common practice. The 2002 Work Safety Law and 2007 Emergency Response Law both include hazard monitoring as a basic system.

Researchers in social sciences have explored the unpredictable, invisible hazards in human-induced risks in order to develop intuitive measurement models. In political science, the political system risk is simplified into the relationship between several variables; in economics, finance and trade risks are frequently assessed; in sociology, a variety of early warning indicators have been developed, and on this basis, the use of "social indicators" has gained ground.

Generally, whether it is the identification of tangible, visible hazards or the prediction of intangible, invisible man-made hazards, the core of traditional methods includes two aspects: one is the type of risk, i.e. which factors may cause harmful consequences; the other is the level of risk, i.e. the probability of such harmful consequences. A general assumption about risk type is that all kinds of risk exist in a stable form, and can be accurately identified with in-depth surveys; and a general assumption about risk level is that risk is measurable, and with appropriate methods, the level of risk can be accurately described. But both assumptions are now facing challenges.

First, few risks stay unchanged; instead, they often have a "ripple effect". Therefore, natural disaster researchers have developed the "disaster chain" approach and put forward four "disaster chains" in China: (1) (typhoon)-rainstorm-disaster avoidance-mudslide-flood-farmland, road and building damage-drowning deaths, etc.; (2) earthquake-(rain and snow)-collapse-sand surge-damage to buildings (housing, factories, lifeline)-transportation disruptions-communications disruptions-poison gas, fire, etc.; (3) drought-dust storm-heat wave-water shortage-damage to crops, 
forests and grasses-shrouded rural and urban communities ${ }^{41}$; and (4) cold wave-snow disaster (frost, cold temperature)-biological effects of cold and frost (mechanical failure, structural damage, damage to agricultural and pastoral areas. ${ }^{42}$ This approach has the following shortcomings: the "disaster chain" only considers natural factors while overlooking social factors, but risk is embedded in social fabric and obviously socially constructed, and the impact of social factors can change the path of the "disaster chain" and the consequences of disaster, resulting in more complicated forms. Moreover, although the "disaster chain" theory believes risks are changing rather than in stable form, its study of risk dynamics still follows a linear pattern. Judging from the reality and future trends, risk is becoming more and more nonlinear. Demography, the environment, technology and socio-economic structure have transformed traditional risk, but have given risen to new risks, changed the vulnerability of populations to risk, and transformed how impact spreads, leading to some kind of comprehensive impact. ${ }^{43}$

Second, risk is becoming more difficult to measure. In traditional risk measurement models, actuaries of insurance companies usually use loss frequency and degree as well as expected value and standard deviation of loss in a given period of time to measure loss probability distribution. Loss frequency is defined as the number of loss occurrences over a given time interval. If historical data of risk units are available, the probability of loss per risk unit can be estimated by dividing the number of risk units into loss frequency; and the degree of loss represents the scale of loss caused by an incident. ${ }^{44}$ Now, risk has become a general term and essentially unmeasurable. According to Beck, nuclear risk, chemical product risk, genetic engineering risk and ecological calamity risk have completely destroyed the four pillars of risk calculation. ${ }^{45}$ Specifically, first, these catastrophes often cause irreparable global damage that makes monetary compensation in risk calculation meaningless; second, in the worst-case scenario, the safety concepts endowed by risk calculation, including damage control and early-warning and monitoring of the consequences, are also meaningless; third, the spatial-temporal scopes and boundaries of disaster disappear completely, making calculation impossible; finally, the impact of disaster no longer has a conventional form, so risk calculation would have no result. Certainly, this does not mean that researchers should abandon risk calculation altogether. Anyway, risk calculation is always better than nothing, as long as we do not blindly believe the results of risk calculation or take it as the only standard of risk management.

\footnotetext{
${ }^{41}$ Ma Zongxiang. Reflections on Natural Disaster Response in China. China Emergency Management, 2007 (2).

${ }^{42}$ Shi Peijun. Theory and dynamics of disaster research. Journal of Natural Disasters, 2002 (3).

${ }^{43}$ OECD. Emerging Risks in the 21st Century: An Agenda for Action. 2003.

${ }^{44}$ Scott E. Hanigton \& Gregory R. Niehaus. Risk Management and Insurance (2nd edition; trans. Chen Bingzheng, Wang Jun \& Zhou Fuping), Beijing: Tsinghua University Press, 2005, p. 42.

${ }^{45}$ Ulrich Beck. "Survival Issues, Social Structure, and Ecological Enlightenment", in Ecological Enlightenment: Essays on the Politics of the Risk Society. NJ: Humanities Press, 1995, p. 23.
} 
Since risk is socially constructed, it is not enough to simply define risk by type and level. Researchers in psychology have developed measurement methods to assess how different types and levels of risk are perceived by people. The most representative approach is quantitative risk perception of Paul Slovic.

How to deal with disasters has become a core issue in the field of disaster research. Three branches of management have responded differently: researchers in managerial science and engineering try to answer some specific technical questions, such as the optimal algorithm for emergency facilities site selection and emergency resources dispatch; business management researchers work to provide effective management tools for enterprises, such as how to deal with operational difficulties as a result of product quality defects; and public administration researchers seek to provide the government with optimal institutional design, such as disaster response regulations. The core of disaster response is coordination, and the prerequisite of coordination is consensus on issues among different types and levels of organizational actors. Distributed cognition is such a methodological exploration. As a "social-technical" approach, it demonstrates a systematic effort to improve the structured learning process to support decision makers in taking coordinated action in the face of threats. It is an interdisciplinary task in itself, in which anthropology extends cognition beyond individuals, psychology explores the cognitive process by giving meaning, network analysis provides methods for understanding cognitive structures in complex environments, and computer science provides technological means for computational simulation of complicated information exchange between networks. ${ }^{46}$ To answer the question of what optimal emergency structure is, a set of emergency performance evaluation methods must be developed. Based on case studies and quantitative evaluation, a high-quality case database should be established and emergency performance of each case be calculated in order to compare the performance of different emergency structures. As to what optimal resource allocation is, research methods must be introduced to work out optimal resource allocation schemes. It goes without saying that resource allocation is not just a technical issue, but needs to bring in research approaches of political science and public policy.

Then how to integrate risk research and disaster research methods? On the one hand, risk identification makes disaster response possible, as the type and level of risk defined through risk identification provides the basis for disaster response; on the other hand, disaster response must be based on risk identification, because both optimal structure and optimal resource allocation of disaster response can be boiled down to "ability" which is a relative concept based on specific risk types and risk levels. It is not advisable to require communities to have the ability to cope with catastrophes, or provincial governments to deal only with ordinary disasters. Combining

\footnotetext{
${ }^{46}$ Louise Comfort. "Distributed Cognition: The Basis for Coordinated Action in Dynamic Environments". Conference Paper, 2008.
} 
risk research with disaster research makes the calculation of value at risk meaningless. ${ }^{47}$ Instead of judging the best time for intervention by calculating value at risk, we are now trying to deal with risk through building emergency response capacity.

The main purpose of crisis research is dissemination and communication, so we need to establish the means of dissemination and the discourse system, especially those that fit the Internet era. To this end, research methods need to be updated, and research literature should go beyond books, papers, objects and other traditional materials; analysis of Internet resources should be encouraged, and Internet-based methods should be developed accordingly.

\subsubsection{Interdisciplinary Approaches and Techniques}

System theory argues against examining a social phenomenon in isolation and advocates a holistic and systematic approach from the beginning. In 1972, the Club of Rome published The Limits to Growth, a research report written by Dennis Meadows and other young scientists. They used system dynamics models and high-speed computers to simulate the complicated relationships among twelve factors, including demography, energy, the environment, water, health and economic development. On this basis, the report predicts that the world systems may collapse because of the energy crisis, and growth may come to a halt in 2100 . This is a prediction of catastrophe for mankind as a whole and has had significant impacts worldwide. Ilya Prigogine's dissipative structure theory is based on uncertainty. It holds that an open system can absorb negative entropy flow from the outside by exchanging materials and energy, thus reducing its own entropy and forming a far-from-equilibrium orderly structure called dissipative structure. French mathematician Rene Thom created catastrophe theory to describe the stable and unstable states of a system. Social scientists should borrow the dissipative structure theory and catastrophe theory to study the catastrophe and collapse of the complex system of society.

Systems theory and information theory use the concept of entropy to represent the degree of disorder of a system: the greater the disorder, the higher the entropy. They also use entropy to define the amount of information, and put forward that the amount of information is negative entropy. When the system acquires information, disorder, or entropy, decreases; the more information acquired, the greater decrease in disorder (entropy); and a decrease in system disorder (entropy) means an increase in order and organization of the system. In the social science study of disaster, risk means the possibility and uncertainty of loss, and crisis is the loss after such possibility and uncertainty become reality. Loss, in essence, is a disturbance to the order of social system and people's normal lives. Therefore, the social science study of disaster will benefit a lot from borrowing the concept of entropy and relevant knowledge from natural sciences.

\footnotetext{
${ }^{47}$ This is also a bottleneck that has existed for long in traditional risk early warning research.
} 
By comparison, the two approaches have their own advantages and disadvantages. The former has direct explanatory power over particular issues but cannot meet the requirements for transdisciplinary research. The latter has universal explanatory power over general issues and is transdisciplinary per se, but is too abstract. Therefore, how to make the former applicable to general issues and the latter to particular issues is a main task of methodological innovation in the social science study of disaster. 